\title{
Fun and efficiency of the Wii balance interface
}

\section{Wim Fikkert*, Niek Hoeijmakers, Paul van der Vet and Anton Nijholt}

\author{
Department of Computer Science, \\ Faculty of EEMCS, \\ University of Twente, \\ P.O. Box 217, Enschede 7500 AE, The Netherlands \\ Fax: +31534893503 \\ E-mail: f.w.fikkert@utwente.nl \\ E-mail: n.j.hoeijmakers@student.utwente.nl \\ E-mail: p.e.vandervet@utwente.nl \\ E-mail: a.nijholt@utwente.nl \\ *Corresponding author
}

\begin{abstract}
Balance input in human-computer interfaces can be beneficial, enjoyable and entertaining. Users can navigate a virtual (game) world or an (empirical) dataset while having their hands free to issue other commands. A new low-cost balance interface, the Nintendo balance board, offers widespread use of this form of input. We performed two experiments that explore the fun and efficiency of this interface for navigating simple virtual worlds. Our first study showed that the users completed $2 \mathrm{D}$ navigation tasks slower with the balance input method. The second study showed that a standard self-centreing joystick outperforms balance-based interfaces. However, the users in both studies enjoyed the balance input methods despite it being less efficient. The board interfaces performed similarly for easier navigation tasks; leaving the hands free for manipulation tasks while coarse navigation can be performed by the shifting body mass.
\end{abstract}

Keywords: user interfaces; evaluation; input devices and strategies.

Reference to this paper should be made as follows: Fikkert, W., Hoeijmakers, N., van der Vet, P. and Nijholt, A. (2010) 'Fun and efficiency of the Wii balance interface', Int. J. Arts and Technology, Vol. 3, No. 4, pp.357-373.

Biographical notes: Wim Fikkert received a MSc in Computer Science at the University of Twente and is now a PhD student in the Human Media Interaction Group in the Department of Computer Science at the University of Twente. His research focuses on natural forms of interaction with a current focus on gesturebased and multi-touch interactions.

Niek Hoeijmakers is a graduate student in the Human Media Interaction Group at the Department of Computer Science in the University of Twente. He received a BSc in Computer Science at the University of Twente.

Paul van der Vet is an Assistant Professor in the Human Media Interaction Group in the Department of Computer Science, at the University of Twente. 
His background is in chemistry and philosophy of science. Currently, he is working on interaction with bioinformatics tools and systems and on interactive visualisations.

Anton Nijholt received a MSc in Mathematics and Computer Science (1974) at Delft University of Technology and a $\mathrm{PhD}$ in Computer Science at the Vrije Universiteit Amsterdam. He has held positions at various universities in Canada, Belgium and The Netherlands. Since 1989, he is a Full Professor for Computer Science at the University of Twente (Enschede, The Netherlands). $\mathrm{He}$ is now the Head of the Human Media Interaction Research Group in the Department of Computer Science of the University of Twente. His recent interests include entertainment computing, multimodal interaction and brain-computer interfacing.

\section{Introduction}

Interacting with virtual worlds can be made increasingly immersive by adding modalities for input and output to that interaction (Oviatt, 1999). New forms of interaction that are based on such added input and output modalities increasing flood the consumer market, introducing engaging and often unexpected new experiences to the public. Hand and other bodily movements now serve as input for game consoles (Fikkert et al., 2007). Similarly, input that is based on body gait and centre of mass in human-computer interfaces can benefit the interaction by enabling natural proprioception. When using these types of input for navigation tasks in the virtual world, users will have their hands free to perform other, tasks such as issuing commands to a virtual game world (World of Warcraft, Killzone) at the same time, for example, through speech or with the hands. Such interfaces have the potential to be more immersive, efficient and - perhaps most importantly enjoyable because users can divide attention between tasks. In addition, whole body input can be highly entertaining even to the point where it entices users to start interacting (Reidsma et al., 2006). This is, for example, illustrated by Nintendo's Wii Fit game that is enthusiastically used in elderly homes for entertainment and in medical centres for rehabilitation. ${ }^{1}$

Our contribution is an evaluation, based on two experiments (Sections 3 and 4), of the performance and enjoyability of performing navigation tasks in a virtual world by shifting the centre of body mass. Section 2 describes related work on bodily computer interactions and ways to evaluate them. In Section 3, our first experiment explores centre of body mass path tracing while simultaneously issuing simple commands. We explored factors such as task completion time, error rates and enjoyability of the Nintendo balance board interface that was combined with and without a Nintendo Wiimote. Our second experiment, see Section 4, evaluated navigation methods based on shifting the centre of body mass with a Fitts' law-based tapping test. Efficiency, error rates and subjective ratings on, among others, fatigue, effort and operation speed are reported on two variations of the Nintendo balance board interface and other, more familiar interfaces that are in wide use for navigation tasks, for example, mouse and joystick. This paper is concluded in Section 5 with a discussion on our findings and with an assessment of how navigation based on shifting the centre of (body) mass can be applied. 


\section{Related work}

\subsection{Whole body input}

Navigating virtual worlds through virtual walking, where the user can physically walk freely through an entire world, have been addressed extensively in systems that fully immerse their users. The VirtuSphere ${ }^{2}$ enables virtual walking by placing its users inside a large sphere in which they can walk, run, roll and even jump. See Kruijf's $\mathrm{PhD}$ thesis for a more extensive overview (Kruijff, 2006). Beckhaus et al. (2005) evaluated a Dance Pad with six directional buttons with their chair-based travel interface. Both devices are a balance-based interface with the Dance Pad as a low-cost solution for simple navigation tasks. The chair interface was very intuitive and provides a large potential for additional tasks, however, it was much more expensive that the Dance Pad device. Antle et al. (2009) built Springboard, a interactive environment prototype, with which users can explore concepts of social justice through embodied interaction. The Springboard itself is a crib mattress spring so that the users' nature state of balance in standing is upset when standing on the Springboard. The CyberCarpet enables two-dimensional locomotion on an infinite plane by letting a user walk on an array of balls that are actuated by a belt on a turntable (Schwaiger et al., 2007). Users were fully immersed in an entire virtual city by wearing a head-mounted display helmet.

Interfaces that do not completely immerse their users typically require them to remain stationary for large displays or use users' mobility in portable interfaces. The JoyFoot uses accelerometers to detect ankle movements for controlling navigation in a virtual world (Barrera et al., 2004). The JoyFoot was evaluated with a large display in a game setting where users had to navigate an asteroid debris field game world in two dimensions by moving their feet on the floor. After a brief training period, all users could navigate in the JoyFoot game. A portable solution was presented in Paelke et al. (2004) where a PDA equipped with an external camera was used to play an augmented soccer game with the user's foot.

Reidsma et al. (2006) enticed users to engage in a dancing dialogue with their virtual dancer. A game dance mat ${ }^{3}$ is used to detect the user's presence while a crude computer vision solution is used to detect dancing motions. Map manipulation has also been shown to benefit from combining a touch-sensitive wall-sized display for selection and activation with centre of body mass input from a Nintendo balance board for navigation (Schöning and Krüger, 2008). It has also been suggested that the balance board can be a useful modality to perform control tasks by the feet on one degree of freedom (de Haan et al., 2008). Our work investigates if such control tasks can also span multiple degrees of freedom.

\subsection{Evaluating input methods}

Methods for input in human-computer interaction dialogue can be thoroughly evaluated on task completion times, error rates and user satisfaction on various tasks using the ISO 9241-9 standard (ISO, 2000) for evaluating non-keyboard devices. This standard is based on Fitts' Law for human movements (Fitts, 1954). The standard defines performance measures for evaluating input devices or techniques using basic interfaces tasks such as tap, drag and trace. Like Schöning and Krüger (2008), we are interested to explore the effects of shifting the centre of body mass for input on path tracing tasks, for example, as found in map navigation.

The underlying assumption in Fitts' law is that the cognitive aspects of an interaction are removed to such an extent that the motor abilities of humans are tested. Although, Fitts' 
law focuses on direct pointing tasks it has been shown to hold for indirect pointing tasks as well (MacKenzie, 1991). In addition, Fitts' law-tests primarily target motor control for the human arms yet it has been shown to apply to eye gaze (Zhang and MacKenzie, 2007) and leg-based (Medina et al., 2009) tasks as well. A derivative of Fitts' law is the steering law for trajectory-based human-computer interaction (Accot and Zhai, 1997). This law is derived from Fitts' (1954) law for human movement and it predicts the movement time for steering through a two-dimensional tunnel. We will use Fitts' law in an extensive evaluation of the Nintendo balance board interface in Section 4. The evaluation in this section is of an exploratory nature that is why we chose to simplify the evaluation method slightly.

\section{Path tracing}

In our first experiment, we set out to explore path tracing with simultaneous command issuing. Our goal was to assess performance and enjoyability of path tracing based on shifting the body's centre of mass while shifting their attention back and forth between the task of path tracing and issuing commands. Note that the goal of this experiment is to explore this type of interface as it is used in many homes in the form of the Nintendo Wii gaming console. Users performed a time-crucial navigation task in a set of simple mazes by shifting their centre of body mass. While performing this navigation task the users were required to press buttons on a handheld device. These commands were issued at predefined locations in the maze, without that the user could not continue.

\subsection{Method}

Our setup was a large display interface on which a set of simple mazes were depicted (see Figure 1). Two interfaces were compared that were used to navigate through the maze. Firstly, subjects navigated by shifting their body's centre of mass while pressing buttons on a handheld device. Secondly, the handheld device was used for navigation as well. We hypothesise that the balance board will be more intuitive for simultaneously navigating and issuing commands while not suffering loss in performance.

\subsubsection{Devices for navigating the maze}

A Nintendo Wiimote and Nintendo balance board were used in a within subjects evaluation (see Figure 1). The Wiimote is a handheld device that has three perpendicular accelerometers and an IR camera that detects fixed light sources for absolute pointing (Fikkert et al., 2009). The balance board (or board for short) has four pressure sensors at each of its corners.

Our test subjects completed two scenarios in counterbalanced order. The first scenario (BW) combined the board with a single Wiimote. In BW, the board was used for navigation; the subject stands on the board and shifts its centre of mass to move the avatar. Leaning forward moved the avatar up, left moved it left and so on. By leaning more, the movement speed could be varied from none (when standing perfectly still and centred) to a set maximum that was reached by placing all weight on the corresponding pressure sensor. This is very similar to how a Segway works. The Wiimote could be held in the preferred hand (either left or right) and on set positions in the maze the subject was required to issue an command with it (see Section 3.1.2). In the second scenario (WM), our subjects only used one Wiimote to control both the avatar and issue the commands in the maze. A similar form of navigating 
Figure 1 The maze navigation task combines the input from a balance board and Wiimote alike (see online version for colours)
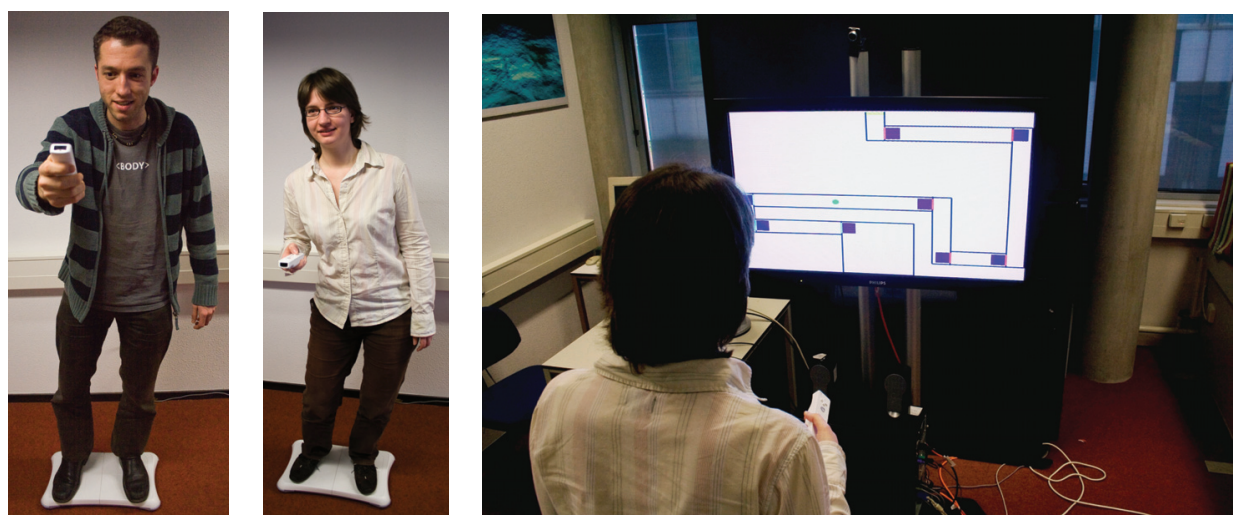

Note: Subjects enjoyed both navigation approaches; using either the Wiimote or balance board. While navigating, users were required to press buttons on the Wiimote in order to complete the maze.

Figure 2 The set of mazes consisted of very simple (a) linear paths to (b) more complex paths (see online version for colours)

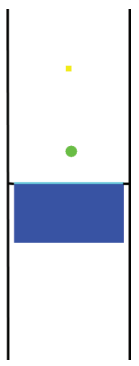

(a)

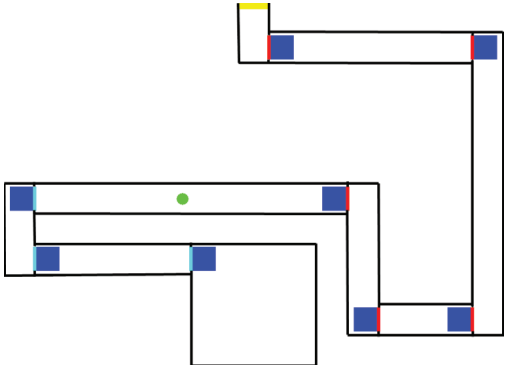

(b)

Note: Maze components were identified by their colour. In all cases, only one path existed.

the maze with the Wiimote was selected compared to BW; the accelerometers were used to detect roll (for moving left and right) and pitch (for moving up and down). Rolling left/right moved the avatar left/right while pitching up/down moved the avatar up/down accordingly. For both devices, we introduced a small threshold below which no movements would occur ( 0.1 of the maximum sensor value). The sensor values ranged from -1 to 1 and we mapped these values, when exceeding the aforementioned threshold, linearly to a maximum movement speed. The avatar maximum movement speed was equal for BW and WM, matching the maximum pitch or lean.

\subsubsection{The maze task}

Subjects in our study navigated an avatar (green dot) through four mazes, seen from above, of varying difficulty (see Figure 2). The four mazes were presented in the same order for both of the above scenarios. Before starting the first maze, our subjects were allowed brief training session to get used to the device for navigation and to learn how to issue 
commands with the Wiimote. Each maze consisted out of a sequence of rooms that was connected by doors, ending with a maze exit. The subject had to navigate/walk to a each (closed) door and, while standing in front of the door on a doormat, press a button to open the door. The button to press was displayed on-screen only when a subject was standing on a doormat. After a correct button-press, the door would open so that the subject could proceed to the next room. Pressing an incorrect button would give a visual error signal.

\subsubsection{User evaluation}

For each maze, we measured the task completion time from the onset of each maze till the time it finished. This timespan was taken as a measure for performance in addition to the time that subjects stood still on doormats. As a measure for error rate, we counted impacts with the walls of the maze and with closed doors in addition to incorrectly entered commands. Also, we measured the number of times that a subject drifted off a doormat in front of a closed door and the time it took to reposition the avatar on the doormat. User personal data were gathered to assess their previous experience with the devices in this experiment or with similar input devices. After completing each scenario, the user filled out a questionnaire in which they rated the interaction on topics such as overall intuitiveness, the accuracy and smoothness of the avatar movements and on fatigue in arms, legs and hands. We explicitly split questions on intuitiveness from ease of use to remove the bias introduced by the subjective interpretation of these terms. The questionnaire was taken from the ISO 9241-9 standard (ISO, 2000). Upon completing both scenarios the users rated which device they enjoyed the most for navigating.

\subsection{Results}

About 18 subjects participated in our evaluation, 4 female and 14 male $(\mu=29$ years, $\sigma=11$ years, ranging 18-56 years). Nine subjects held a Masters degree, six a Bachelors and three had no degree. On a 1-5 Likert-style scale that varied from no experience to daily use, our subjects were proficient with the Wiimote $(\mu=3.4, \sigma=1.2)$ before taking part in the study but the balance board $(\mu=2.3, \sigma=0.9)$ and other motion capture solutions ( $\mu=2.0, \sigma=0.8$ ) were less familiar. The complete test with both scenarios was completed on average in $9 \min (\sigma=2$ minutes).

Normal distributions for our questionnaire data and for all interface trials were found using one-sample Kolmogorov-Smirnov (K-S) tests. The WM and BW scenarios were compared with a paired-samples $t$-test. With respect to performance, we found that task completion time with WM was significantly faster (17 sec faster, $p=0.04, t=2.208$ ). After the test, most subjects (ten for WM and four for BW) also indicated that they experienced WM to be the faster solution for navigation. The number of wall and door hits did not differ significantly between BW and WM, nor did the number of incorrectly issued commands. However, 17 subjects indicated after the test that they experienced WM to be less prone to bumping into walls or doors (1 subject experienced no difference). We found that our subjects maintained their location on doormats more consistently for WM ( $\mu=4.3$ fewer drifts, $p<0.01, t=3.725)$. A drift is defined as moving off and back on a doormat. The total time spent on doormats did not differ significantly but on average each pass took significantly less time using BW ( $\mu=0.47 \mathrm{sec}$ faster, $p=0.04, t=-2.190)$. 
Table 1 Measurements of maze task

\begin{tabular}{lcc}
\hline & $B W$ & $W M$ \\
\hline Task completion time & $0: 02: 16,43(0: 00: 26,08)$ & $0: 01: 58,99(0: 00: 25,27)$ \\
Wall hits & $47.6(6.9)$ & $50.9(15.0)$ \\
Door hits & $13.2(6.0)$ & $12.5(16.7)$ \\
Incorrect keys & $33.3(43.6)$ & $26.7(23.1)$ \\
\hline
\end{tabular}

Note: Task completion time in h:mm:ss, ms, other results are counts. Means are reported, $\sigma$ between parentheses $(N=144)$

Table 2 Questionnaire means per interface $(N=36), \sigma$ between parentheses

\begin{tabular}{lcr}
\hline & $B W$ & $W M$ \\
\hline Enjoyability of the game & $3.5(1.1)$ & $3.3(1.4)$ \\
Arm fatigue & $4.9(0.3)$ & $4.5(0.6)$ \\
Standing fatigue & $4.4(1.0)$ & $4.8(0.4)$ \\
Accurate avatar movements & $3.2(0.7)$ & $3.9(0.8)$ \\
Intuitive avatar movements & $3.9(1.0)$ & $3.4(1.0)$ \\
Learning to move avatar & $3.9(1.2)$ & $4.1(0.9)$ \\
Smooth avatar movements & $3.5(0.9)$ & $3.5(1.0)$ \\
Easy to open doors & $3.3(1.1)$ & $3.6(0.9)$ \\
\hline
\end{tabular}

Note: Scale $1-5$, higher is better.

There was no significant difference in the task completion time between BW and WM (see Table 1). Likewise, we did not find significant differences between the number of hits into walls and doors nor in entering incorrect keys to open a door.

We found no significant difference in ease of learning, intuitiveness of the navigation and smoothness of the navigation between both techniques (BW and WM) for navigation in our user opinions after each scenario. Table 2 presents these results. However, in the evaluation after the test most subjects (ten) indicated that BW was easiest to learn while six subjects found WM easier to learn. Likewise, BW was considered the most intuitive (13 subjects, 2 for WM). Our subjects rated the navigation accuracy significantly higher in favour of WM ( $p<0.01, t=-3.198)$ after each scenario and in the evaluation after the test (12 subjects, 2 for BW). Although we did not find a significant difference for opening the doors between BW and WM, five subjects indicated that they were not familiar enough with the Wiimote to find the required button right away. Although there was no significant difference in the extent that our subjects enjoyed using either WM or BW, they indicated after the test that BW was the most fun (12 subjects).

In observations during our trials, we note that most subjects navigated in only one direction with WM at any one time. For example, a subject would move up first and then turn left rather than moving in both directions simultaneously. Similarly, we observed that our subjects would in most cases complete the navigation task first. Only after ensuring that they remained stationary on a doormat would they focus on opening the door. In addition, it was observed that most subjects would split the navigation and command tasks implicitly by first moving the avatar to the correct location and then focusing on the command that would open the door. During the trials four subjects commented that they found our implementation for moving up and down with WM counter intuitive. They would rather invert it so that pitch down moves the avatar forward, arguing that would be exactly the same as the BW implementation. 


\subsection{Discussion}

By using the body's centre of mass to navigate a virtual world users keep their hands free to complete to perform other tasks simultaneously such as issuing commands. We have compared the Nintendo balance board to a handheld Wiimote for navigating a series of simple mazes. While navigating a maze of consecutive rooms, subjects opened doors between these rooms on set locations in front of the doors. Users could navigate with the board by shifting their centre of body mass through leaning or by changing the roll and pitch of the Wiimote.

We hypothesised that the balance board would be more intuitive for the navigation task while not suffering a performance loss. Although, we found that the board was somewhat easier to learn and use, the Wiimote was significantly faster in navigating the maze without resulting in an increase in navigation errors or errors in the issued commands. In addition, subjects found it harder to maintain a steady location with the board which is demonstrated by the number of corrections that were made in the BW scenario for maintaining the position on a doormat. This suggests that using the same input modality (a handheld device) performs better for navigating a virtual world while simultaneously issuing commands. However, we argue that the fun factor of an interface is equally or even more important for many applications, especially games. Also, easier, coarse navigation tasks might be adequately performed with an interface based on shifting their balance.

Our subjects strongly indicated that they enjoyed using the combination of Wiimote and the balance board more, although it was not faster to complete the game. This raises a good point in that novelty is a very important factor for the perceived fun factor. We expect that a comparison between Wiimote and joystick would provide similar differences. A truly unbiased assessment requires the users to become much more proficient with both devices for which extensive training is required (Goonetilleke et al., 2009).

Four of our subjects wanted to invert the pitch for navigating to match balance board navigation; leaning forward would move the avatar forward. We chose not to implement that approach in favour of mimicking ray-casting to the screen where pointing to the bottom of the screen would move the avatar down.

\section{Fitts' law comparison}

Having evaluated simultaneous path tracing and command issuing we set out to explore and evaluate the performance of two different ways of mapping the body's centre of mass to a location on the screen. To evaluate these two interface implementations based on performance, we performed a Fitts' law-based tapping test that focuses on the motor abilities of the user. The Wiimote interface that was used in our path tracing test is included in this evaluation as well as a self-centreing joystick and mouse that represent best practice approaches. We hypothesise that these best practice methods will outperform the board interfaces in terms of throughput but not on the amount of errors made. In addition, we expect that the board interfaces will be more enjoyable.

\subsection{Method}

Five interface implementations that indirectly control a cursor on a large display (see Figure 1), were compared. These five interface implementations were a standard doublebutton mouse, a self-centreing joystick, a Wiimote and two implementations of a Nintendo balance board that detects the user's centre of body mass. 


\subsubsection{Tapping interfaces}

Two interfaces were implemented with the Nintendo balance board. The first interface, 'board (abs)', uses absolute positioning by mapping leaning forward to the upper edge of the display $(y=0)$, leaning left to the left edge $(x=0)$, leaning right to the right edge $(x=1)$ and leaning back to the lower display edge $(y=1)$. The second interface, 'board (add)', uses additive positioning of the cursor. By leaning slightly left, the cursor would move left (negative $x$-direction). Movement speed was matched to the extent of leaning. We included two types of balance-based interaction, board (add) and board (abs), because the board (add) was used in the first (maze) experiment. Our users there noted that it is was hard to move the avatar through the maze with great accuracy. The board (abs) interface is based on that of a self-centreing joystick. For the Wiimote, its accelerometers were used to detect pitching and rolling. Pitching upwards would move the cursor in the negative $y$-direction, that is, upwards, while rolling right moved the cursor in the positive $x$-direction, that is, right. We applied the same sensor threshold below which no movements would occur ( 0.1 of the maximum sensor value) as in the path tracing experiment, except for the mouse of course.

The same large display was used as in our maze task (see Figure 1), and subjects stood at a distance of $2 \mathrm{~m}$ from this display. As the balance board lacks a button to click with, a Wiimote was handheld so that the subjects had a button (the 'A') to press. In the Wiimote setting, the users also pressed the 'A' button to click. The mouse and joystick were placed on a metre high stand.

\subsubsection{Tapping task}

A point-and-click task based on Fitts' (1954) Law was used to compared the five interfaces based on throughput and error rates. Although Fitts tested only direct pointing in his work, MacKenzie (1991) showed that Fitts' law also applies to indirect pointing tasks, which occur in $\mathrm{HCI}$ in, for example, the form of mouse-based pointing. We followed the guidelines set out by Soukoreff and MacKenzie (2004) for this tapping test. In this test, users were required to repetitively tap between two targets. The speed in which they tap indicates the throughput, or index of performance (IP) in bits/s, of the device or interface that they use. With an increase in distance $d$ between targets and a decrease in target size $w$ along the axis of movement, the task difficulty, which is defined by the index of difficulty (ID) in bits, is increased. Even though Soukoreff and MacKenzie (2004) argue to use a bit-range from 2 to 8 bits, we used an ID range from 1 to 4 bits because that better represents the maze task on a large display that we described in Section 3. The ID range was constructed based on the display size (see Table 3). Each trial (combination of $d$ and $w$ ) was repeated 20 times, following Soukoreff and MacKenzie (2004). Subjects tapped 20 times for each ID value in both horizontal and vertical directions. The order of these tasks were randomised per movement direction. The order of the two movement directions were also randomised.

The task completion time of each tap task was stored. Each tap is defined from the onset of movement to the button-down event on-target (Isokoski, 2006). Errors in clicking were only filtered when a double-click occurred on the location of the previous tap-task. We calculated the effective target width by taking $w_{e}=4.133 \sigma$. This makes $w_{e}$ the central $96 \%$ of the spatial distribution of the user's clicks under the assumption of a normal distribution. The effective index of difficulty was calculated by taking $\operatorname{ID}_{e}=\log _{2}\left(\left(d+w_{e}\right) / w_{e}\right)$ (MacKenzie, 1991).

Interface ratings on subjective qualities such as intuitiveness, accuracy and speed were gathered in a questionnaire based on ISO (2000, see Appendix C). We expanded this questionnaire slightly with questions targeting fatigue in the lower and upper legs in addition to fatigue from standing upright during the entire test. 
Table 3 The range of ID used in both horizontal and vertical directions

\begin{tabular}{lcc}
\hline$d(\%)$ & $w(\%)$ & ID(bits) \\
\hline 0.80 & 0.15 & 2.66 \\
0.80 & 0.10 & 3.17 \\
0.60 & 0.25 & 1.77 \\
0.60 & 0.15 & 2.32 \\
0.60 & 0.05 & 3.70 \\
0.40 & 0.10 & 2.32 \\
0.40 & 0.05 & 3.17 \\
\hline
\end{tabular}

Source: MacKenzie (1991).

Note: The distance $d$ and target size $w$ form the ID as follows: ID $=\log _{2}((d+w) / w)$.

The display size was $93 \times 52.5 \mathrm{~cm}$.

\subsection{Results}

About 12 subjects took part in this within-subjects test. Subjects were male, righthanded and 29 years old on average (ranging 25-47 years, $\sigma=6$ years). Eight subjects held a Masters degree and four held a Bachelors degree. The whole test with the five interfaces was completed in $61 \min (\sigma=9 \mathrm{~min})$. Subjects scored high on computer competence $(\mu=6.6, \sigma=0.7)$ and were moderately familiar with the Wii game console $(\mu=4.7, \sigma=1.4)$. They were neither very experienced with the Nintendo balance board $(\mu=3.3, \sigma=2.0)$ nor with dance mats $(\mu=2.8, \sigma=2.1)$. Our subjects were unfamiliar with other feet-based interfaces.

A one-sample K-S test showed normal distributions of our questionnaire data. An oneway ANOVA and REGWQ post hoc test showed significant differences in questionnaire scores on how the interfaces were experienced (see Table 4 for the raw data). We report the most noteworthy results here. The mouse and joystick score higher on general intuitiveness while the board (add) interface scored poorest. The mouse, board (abs) and the joystick were found smoother to operate while board (add) required more effort to operate and was less accurate for pointing than the other four interfaces. The mouse and joystick scored highest on operation speed and overall operation. The Wiimote, mouse and joystick were the most comfortable in their use. Fatigue was mostly felt in the lower legs when operating using either of the balance board interfaces while the mouse, joystick and Wiimote were more fatiguing for the wrist. Users did not report fatigue or discomfort from having to stand during the entire test.

Table 4 Questionnaire results per interface $(N=12), \sigma$ between parentheses

\begin{tabular}{lccccc}
\hline & Mouse & Wiimote & Board (abs) & Board (add) & Joystick \\
\hline General intuitiveness & $6.8(0.5)$ & $4.9(1.1)$ & $5.0(1.3)$ & $4.3(1.8)$ & $6.0(1.2)$ \\
Smoothness & $5.9(0.8)$ & $4.1(1.1)$ & $4.8(1.4)$ & $3.8(1.5)$ & $5.1(1.4)$ \\
Effort required to operate & $5.3(1.2)$ & $4.2(1.2)$ & $3.9(1.3)$ & $2.3(1.2)$ & $4.6(1.5)$ \\
Pointing accuracy & $6.4(1.2)$ & $4.2(1.1)$ & $3.8(1.6)$ & $2.3(1.4)$ & $4.8(1.0)$ \\
Operation speed & $6.0(1.0)$ & $3.9(1.2)$ & $4.3(1.5)$ & $3.4(1.7)$ & $5.2(1.3)$ \\
General comfort & $4.5(1.6)$ & $3.7(1.1)$ & $3.4(1.2)$ & $3.0(1.4)$ & $4.9(1.4)$ \\
Overall operation & $6.2(1.1)$ & $4.5(1.0)$ & $4.3(1.3)$ & $2.9(1.5)$ & $5.4(1.4)$ \\
\hline
\end{tabular}


Using a one-sample K-S test, we found a normal distribution of our trials data for the joystick, mouse and Wiimote. For both balance board cases, however, we found nonnormal distributions for MT ( $Z=1.407, p=0.04)$. It has been shown by Wobbrock et al. (2008) that the effective width $w_{e}$ calculation by MacKenzie (1991) still holds. With a Kruskal-Wallis $\mathrm{H}$ test ${ }^{4}$ we found significant differences between the interfaces for $I P_{e}\left(\chi^{2}=574.938, p<0.01\right)$ and error rates $\left(\chi^{2}=163.412, p<0.01\right)$. No large discrepancies between the designed task difficulty (ID) and the effective task difficulty $\left(\mathrm{ID}_{e}\right)$ were found (see Table 5) by design, ID $=2.19 \mathrm{bits}(\sigma=0.76)$. Mann-Whitney $\mathrm{U}$ post hoc tests (see Table 6) on the five interfaces showed that the mouse interface had a significantly higher throughput and significantly lower error rates than all other devices, followed by the joystick interface. The Wiimote interface had a significantly higher throughput than the board (add) and a significantly lower error rate. However, there were no significant differences in throughput $(p=0.44)$ and in error rate $(p=0.11)$ when compared to the

Table 5 Performance ( $\mathrm{IP}_{e}$ ), task difficulty (ID), task completion time (MT) and error rates per $\operatorname{tap}(N=156)$

\begin{tabular}{lcccc}
\hline & \multicolumn{1}{c}{$I P_{e}$} & $I D_{e}$ & $M T$ & Errors \\
\hline Mouse & $3.71(0.66)$ & $3.18(0.50)$ & $0.88(0.19)$ & $0.04(0.06)$ \\
Wiimote & $1.41(0.37)$ & $2.92(0.53)$ & $2.23(0.74)$ & $0.21(0.21)$ \\
Board (abs) & $1.51(0.56)$ & $2.93(0.46)$ & $2.23(0.93)$ & $0.27(0.27)$ \\
Board (add) & $0.78(0.21)$ & $2.80(0.57)$ & $3.77(1.05)$ & $0.29(0.28)$ \\
Joystick & $1.83(0.50)$ & $2.92(0.59)$ & $1.69(0.49)$ & $0.14(0.15)$ \\
\hline
\end{tabular}

Note: Values for $\mathrm{IP}_{e}$ in bits/s, ID $\mathrm{ID}_{e}$ bits, MT in sec. $\sigma$ between parentheses.

Table 6 Mann-Whitney U test results $(N=156)$ for our five interfaces

\begin{tabular}{|c|c|c|c|c|c|c|}
\hline & Wiimote & Mouse & Board (add) & Board (abs) & Joystick & $p$ \\
\hline & 78.62 & 234.38 & & & & $<0.01$ \\
\hline & 222.83 & & 88.17 & & & $<0.01$ \\
\hline & 152.58 & & & 160.42 & & 0.44 \\
\hline & 117.89 & & & & 195.11 & $<0.01$ \\
\hline \multirow{10}{*}{$I P_{e}$} & & 234.50 & 78.50 & & & $<0.01$ \\
\hline & & 233.02 & & 79.98 & & $<0.01$ \\
\hline & & 232.58 & & & 80.42 & $<0.01$ \\
\hline & & & 91.12 & 221.88 & & $<0.01$ \\
\hline & & & 79.73 & & 233.27 & $<0.01$ \\
\hline & & & & 127.37 & 185.63 & $<0.01$ \\
\hline & 201.97 & 111.34 & & & & $<0.01$ \\
\hline & 142.01 & & 170.99 & & & $<0.01$ \\
\hline & 148.34 & & & 164.66 & & 0.11 \\
\hline & 170.92 & & & & 142.08 & $<0.01$ \\
\hline \multirow[t]{6}{*}{ Errors } & & 102.75 & 210.25 & & & $<0.01$ \\
\hline & & 107.06 & & 205.94 & & $<0.01$ \\
\hline & & 121.30 & & & 191.70 & $<0.01$ \\
\hline & & & 162.24 & 150.76 & & 0.26 \\
\hline & & & 184.68 & & 128.32 & $<0.01$ \\
\hline & & & & 178.25 & 134.75 & $<0.01$ \\
\hline
\end{tabular}

Note: Mean ranks are reported for each pairwise comparison. 
board (abs) interface. The board (abs) interface had a significantly higher throughput than the board (add) interface but the error rate was similar $(p=0.26)$.

During the trials, some subjects commented that the movement direction influenced their results. For example, the Wiimote was easier to roll than to pitch due to the physique of the human wrist. We investigated the differences in the trials data with a Kruskal-Wallis $\mathrm{H}$ test and found significant differences in $\mathrm{IP}_{e}$ for board (abs) $(p<0.01)$, board (add) $(p<0.01)$ and Wiimote $(p=0.01)$. With respect to error rates, we found a significant difference for board (abs) $(p=0.02)$. The Wiimote and board (abs) interfaces had a higher throughput for horizontal tapping while board (add) had a higher throughput for vertical tapping (see Figure 3). Subjects also made more tapping errors in the vertical movement direction with the board (abs) interface.

Figure 3 Throughput for the five devices in our Fitts' law tapping test: (a) in horizontal direction and (b) in vertical direction (see online version for colours)

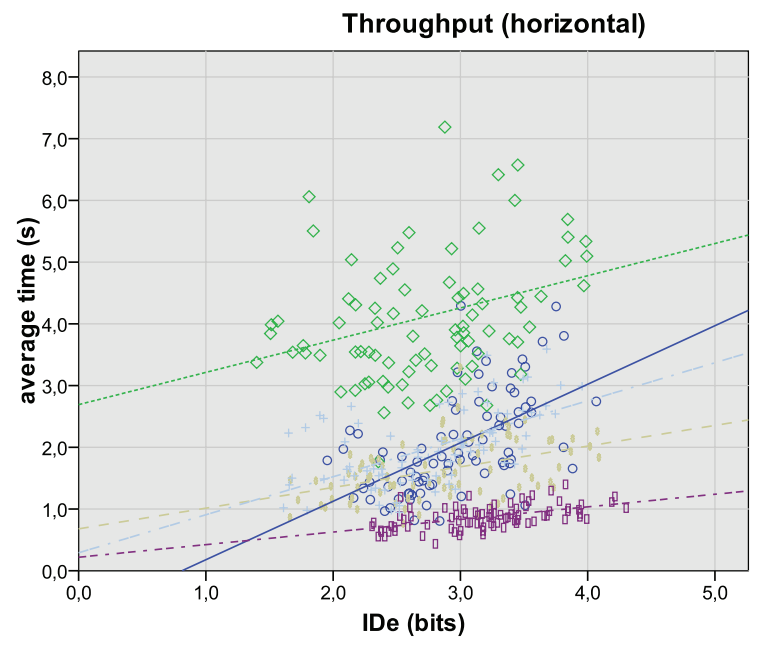

board (abs)

8 board (add)

joystick

mouse

board (abs)

board (add)

joystick

- mouse

wiimote

(a)

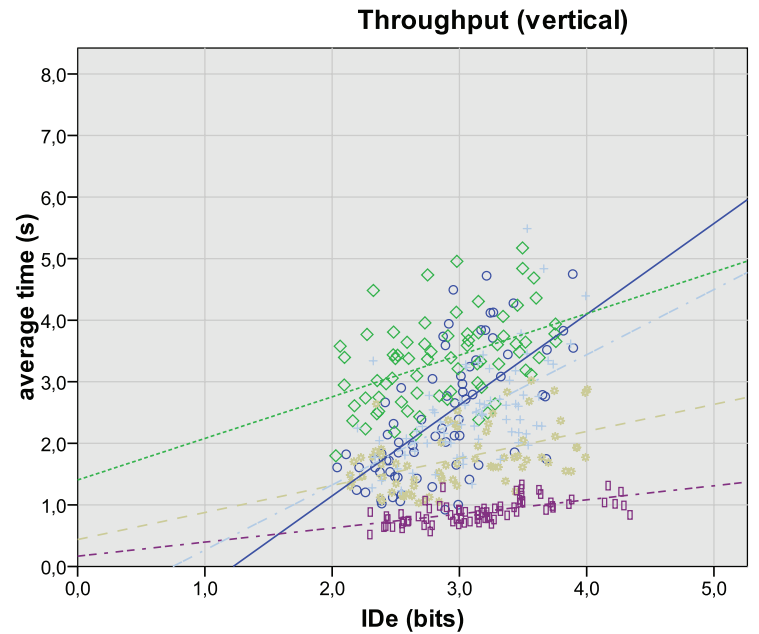

board (abs) board (add)

joystick

mouse

wiimote

board (abs)

board (add)

joystick

mouse

(b) 
When asked to rate the most natural interface, 10 subjects mentioned the mouse while stating that it was most familiar from everyday work. The remaining two subjects mentioned the joystick as the most natural device. Five subjects found the balance board (absolute) the most fun interface while three thought so of the additive case. One subject mentioned the Wiimote as the most fun interface while three subjects mentioned the joystick. The most important reasons for choosing the balance board cases were that it was a new and 'unorthodox' experience and that is required a more active posture while interacting. For both balance board cases, the subjects commented that they felt that clicking with the Wiimote did not add overhead to the task.

During the trials, we observed that three subjects would step forward and backward on the balance board in board (abs) but no so in the board (add) interface where the subjects remained stationary. These three subjects commented that they did so because it made them to reach the extremities of the display with added ease and precision. Users leaned more from side-to-side in the board (abs) interface than they did with the board (add) interface and they mentioned to find it easier to stop the cursor on a target with the board (abs) interface. For the board (add) interface, we observed users move between the two edges of the display while trying to time their taps on the targets that were somewhere along the path that was traveled. The variability visible in Figure 3 for the board (add) interface is expected to be caused by the difficulties our users had to accurately control the cursor location.

\subsection{Discussion}

With a Fitts' law tapping test we compared two indirect navigation methods through shifting the body's centre of mass. The cursor was controlled in an absolute and additive manner by shifting the body while standing on a Nintendo balance board. The absolute method mapped the body's maximum lean to the edges of the display. For comparative reasons, we included a standard mouse, a self-centreing joystick and the Wiimote that were used for navigating the maze task described in Section 3. By repeated reciprocal tapping in a range of task difficulties we measured the throughput and errors rates of these five interfaces.

We hypothesised that the mouse and joystick would outperform the board (abs), board (add) and Wiimote interfaces on throughput but not on error rates while the board interfaces would be the most enjoyable to operate. The mouse was found to have both the highest throughput and the lowest error rate followed by the joystick and Wiimote interfaces as the second and third best, respectively. In addition, the mouse was the most familiar method of controlling the cursor which will explain these results as our users had much more practice with this interface. The mouse's throughput $\mathrm{IP}_{e}$ was, on average, similar to the results of nine ISO 9241-9 studies surveyed by Soukoreff and MacKenzie (2004). We found no difference in error rates between the two balance board interfaces but the absolute way to control the cursor had a significantly higher throughput. However, it was observed that this interface was harder to keep stationary on-target.

Although navigating by shifting the body's centre of mass did not outperform more traditional, best practice interfaces like a joystick, the new interface was thought to be considerable more fun. In addition, the more active posture that was required while interacting was preferred by our subjects. It should be noted that the performance difference for the joystick was especially better for more difficult tasks. Simpler tasks, that is, with an ID under 2.5 bits for the horizontal direction, were performed faster with the board (abs) interface (see Figure 3). The intercept of the trend lines with for board (abs) and board (add) where outside the range defined by Soukoreff and MacKenzie (2004, see pp.758, 759) which we attribute to the difficulties that our subjects had to keep their cursor stationary on a target for the tap-action. 


\section{Conclusions}

Input from the centre of body mass in human-computer interfaces was found beneficial, enjoyable and entertaining in two different experiments that evaluated indirect cursor navigation on a large display. The Nintendo balance board was used as a means to detect shifting the body's centre of mass in these experiments. The first experiment evaluated path tracing performance of two interfaces based on task completion time and error rates. The throughput of the board was lower compared to Wiimote-based navigation although the error rates were comparable. In the second experiment, we evaluated throughput of five interfaces based on Fitts' law tapping tests. This tapping test showed a higher error rate for the board (add) interface that was used in the path tracing task. However, the board (abs) interface had a similar error rate compared to the Wiimote interface. The Wiimote interface had a higher throughput compared to both board interfaces but it was outperformed by a standard mouse and self-centreing joystick in terms of both higher throughput and lower error rate. Because these two devices are more familiar to our users we expect that this has biased the results of our tapping test. A comparison between old and new devices should either facilitate a sufficient training session, see the work of Harada et al. (2009), or such a comparison should balance qualitative results - that are expected to score better for new devices - with efficiency measurements - that are expected to score lower due to less practice. The best solution to this challenge is beyond the scope of this work but this topic merits further research.

The board (abs) interface was easier to position but our subjects found it harder to keep the location stationary as an active posture need to be maintained. Although this active posture inhibits keeping a cursor stationary robustly, our subjects mentioned that they enjoyed this active posture mainly because it was new, fun and unorthodox. It is not surprising that the fun factor is higher for a new interface. We define the fun factor of an interface as a positive influence on its enjoyability, perceived fatigue, operation speed etc. However, this influence is both subjective and hard to measure (Sim et al., 2006). In the work that we present in this paper, the fun factor of the new balance board interface is high but it remains to be seen how long such sentiments lasts. For example, in our tapping test, we found that the Wiimote was not considered as the most fun even though it is also fairly new. We expect that this effect is caused by the high familiarity with the Wii game console with our subjects. It was surprising, however, to find that the more traditional joystick was the most fun interface for a quarter of our subjects. The subjects that chose the joystick interface as the most fun one mentioned that it was usable and familiar. We believe that the usability and frustration in lack of accuracy when using the balance board interfaces were important factors that influenced this choice for our subjects. It was also surprising to find the mouse to be the most 'natural' interface in our tapping test. The extensive practice that most experienced computer users have with this interface lies most likely at the basis of this finding. Although some subjects commented that they would have preferred to invert the Wiimote's pitch control for moving the cursor up and down, none of the subjects in the tapping test thought that this should be so.

Although the performance is lower and error rates are higher for navigation with the body's centre of mass, it does leave the hands free to perform other tasks. Our path tracing test showed no signs that our subjects had trouble to simultaneously press buttons on a hand-held device and navigate by shifting their body's centre of mass. Amazeen et al. (2005) found similar results when users had to switch their attention between their hands 
for bimanual tapping tasks. It was shown in our tapping task that the board (abs) interface performed similarly to its best practice interface - a self-centering joystick - for tasks with low difficulty (ID = [1 - 2.5], (Fitts, 1954)). However, a balance-based interface can be used at the same time as an interface that is controlled by the hands, especially if both interfaces do not interfere in the process of human motor control (Guiard, 1987) and division of attention (Allport et al., 1972). Because the balance-based interface performs similarly for tasks with low difficulty, we expect that an throughput improvement might be attained by combining balance-based interfaces with other interfaces that focus on different input modalities, for example, speech and hand gestures.

\section{Acknowledgements}

This work is part of the BioRange program carried out by the Netherlands Bioinformatics Centre, which is supported by a BSIK grant through the Netherlands Genomics Initiative, and the GATE project, funded by the Netherlands Organization for Scientific Research (NWO) and the Netherlands ICT Research and Innovation Authority (ICT Regie).

\section{References}

Accot, J. and Zhai, S. (1997) 'Beyond Fitts' law: models for trajectory-based HCI tasks', Proceedings of the SIGCHI Conference on Human Factors in Computing Systems (CHI '97), New York, NY, USA: ACM, pp.295-302, ISBN 0-89791-802-9.

Allport, D.A., Antonis, B. and Reynolds, P. (1972) 'On the division of attention: a disproof of the single channel hypothesis’, Vol. 24, No. 2, pp.225-235. ISSN 1747-0218, 11 December 2009.

Amazeen, E., Ringenbach, S. and Amazeen, P. (2005) 'The effects of attention and handedness on coordination dynamics in a bimanual Fitts' law task', Experimental Brain Research, Vol. 164, No. 4, pp.484- 499.

Antle, A., Corness, G. and Droumeva, M. (2009) 'Springboard: exploring embodiment, balance and social justice', CHI EA '09: Proceedings of the 27th International Conference Extended Abstracts on Human Factors in Computing Systems, New York, NY, USA: ACM, pp.3961-3966, ISBN 978-1-60558-247-4.

Barrera, S., Takahashi, H. and Nakajima, M. (2004) 'Joyfoot's Cyber System: a virtual landscape walking interface device for virtual reality applications', International Conference on Cyberworlds, pp.286-292, ISBN 0-7695-2140-1.

Beckhaus, S., Blom, K. and Haringer, M. (2005) 'Intuitive, hands-free travel interfaces for virtual environments', IEEE VR Workshop: New Directions in 3D User Interfaces (VR '05), pp.57-60.

de Haan, G., Griffith, E. and Post, F. (2008) 'Using the Wii balance board' ${ }^{\mathrm{TM}}$ as a low-cost VR interaction device', Proceedings of the 2008 ACM Symposium on Virtual Reality Software and Technology (VRST '08), New York, NY, USA: ACM, pp.289-290, ISBN 978-1-59593-951-7.

Fikkert, W., D’Ambros, M., Bierz, T. and Jankun-Kelly, T.J. (2007) 'Interacting with Visualizations', In A. Kerren, A. Ebert and J. Meyer (Eds.), Human-Centered Visualization Environments, Vol. 4417/2007 of Lecture Notes in Computer Science, GI-Dagstuhl Seminar 3, Heidelberg: Springer Berlin. pp.77-162, ISBN 978-3-540-71948-9.

Fikkert, W., van der Vet, P. and Nijholt, A. (2009) 'Hand-held device evaluation in gesture interfaces', Gesture Workshop 2009, pp.1-2. 
Fitts, P. (1954) 'The information capacity of the human motor system in controlling the amplitude of movement', Journal of Experimental Psychology, Vol. 47, No. 6, pp.381-391, ISSN 0022-1015. Reprinted in Journal of Experimental Psychology, Vol. 121, No. 3, pp.262-269, 1992.

Goonetilleke, R., Hoffmann, E. and Lau, W.C. (2009) 'Pistol shooting accuracy as dependent on experience, eyes being opened and available viewing time', Applied Ergonomics, Vol. 40, No. 3, pp.500-508, ISSN 0003-6870.

Guiard, Y. (1987) 'Asymmetric division of labor in human skilled bimanual action: The kinematic chain as a model', Journal of Motor Behavior, Vol. 19, No. 4, pp.486-517. ISSN 0022-2895.

Harada, S., Wobbrock, J., Malkin, J., Bilmes, J. and Landay, J. (2009) 'Longitudinal study of people learning to use continuous voice-based cursor control', Proceedings of the 27th International Conference on Human Factors in Computing Systems (CHI '09), New York, NY, USA: ACM, pp.347-356.

ISO (2000) 'Ergonomic requirements for office work with visual display terminals (VDTs) Part 9: requirements for non-keyboard input devices', Technical Report ISO/DIS 9241-9:2000, International Organization for Standardization.

Isokoski, P. (2006) 'Variability of throughput in pointing device tests: button-up or button-down?', Proceedings of the 4th Nordic Conference on Human-Computer Interaction (NordiCHI '06), New York, NY, USA: ACM, pp.68-77, ISBN 1-59593-325-5.

Kruijff, E. (2006) 'Unconventional 3D user interfaces for virtual environments', PhD Thesis, Institute for Computer Graphics and Vision, Graz University of Technology, Graz, Austria.

MacKenzie, S. (1991) 'Fitts' law as a performance model in human-computer interaction', PhD Thesis, University of Toronto, Toronto, Ontario, Canada.

Medina, J., Jax, S.A. and Coslett, H.B. (2009) 'Two-component models of reaching: evidence from deafferentation in a Fitts' law task', Neuroscience Letters, Vol. 451, No. 3, pp.222-226, ISSN 0304-3940.

Oviatt, S. (1999) 'Ten myths of multimodal interaction', Communications of the ACM, Vol. 42, No. 11, pp.74-81.

Paelke, V., Reimann, C. and Stichling, D. (2004) 'Foot-based mobile interaction with games', Proceedings of the 2004 ACM SIGCHI International Conference on Advances in Computer Entertainment Technology (ACE '04), New York, NY, USA: ACM, pp.321-324, ISBN 1-58113$882-2$.

Reidsma, D., van Welbergen, H., Poppe, R., Bos, P. and Nijholt, A. (2006) 'Towards Bi-directional Dancing Interaction', In R. Harper, M. Rauterberg and M. Combetto (Eds.), 5th International Conference on Entertainment Computing, Vol. 4161 of Lecture Notes in Computer Science, Cambridge, UK: Springer Verlag, pp.1-12, ISBN 978-3-540-45259-1.

Schöning, J. and Krüger, A. (2008) 'Multi-Modal Navigation through Spatial Information', Proceedings of the 5th International Conference on Geographic Information Science (GIScience '08), pp.151-154.

Schwaiger, M., Thummel, T. and Ulbrich, H. (2007) 'A 2D-Motion Platform: the Cybercarpet', Second Joint World Haptics Conference and Symposium on Haptic Interfaces for Virtual Environment and Teleoperator Systems (WHC '07), pp.415-420, ISBN 0-7695-2738-8.

Sim, G., MacFarlane, S. and Read, J. (2006) 'All work and no play: measuring fun, usability, and learning in software for children', Computers and Education, Vol. 46, No. 3, pp.235-248, ISSN 0360-1315. Virtual Learning?.

Soukoreff, W. and MacKenzie, S. (2004) 'Towards a standard for pointing device evaluation, perspectives on 27 years of Fitts' law research in hci', Int. J. Human-Computer Studies, Vol. 61, No. 6, pp.751-789, ISSN 1071-5819. 
Wobbrock, J., Cutrell, E., Harada, S. and MacKenzie, S. (2008) 'An error model for pointing based on Fitts' law', Proceeding of the Twenty-Sixth Annual SIGCHI Conference on Human Factors in Computing Systems (CHI '08), New York, NY, USA: ACM, pp.1613-1622, ISBN 978-1-60558-011-1.

Zhang, X. and MacKenzie, S. (2007) 'Evaluating Eye Tracking with ISO 9241 - Part 9', HumanComputer Interaction. HCI Intelligent Multimodal Interaction Environments, Vol. 4552/2007 of Lecture Notes in Computer Science, Chapter 85, Heidelberg: Springer, Berlin. pp.779-788. ISBN 978-3-540-73108-5.

\section{Notes}

${ }^{1}$ In USA today, "Wii finds home in retirement communities, medical centers", on 14 may 2008.

2 Available at: http://www.virtusphere.com/, last checked July 2009.

${ }^{3}$ Dance Dance Revolution: http://www.konami.com/ddr/, last checked July 2009.

${ }^{4}$ The Kruskal-Wallis H is the non-parametric equivalent of the one-way ANOVA test. 